\title{
ON VOLUME DISTRIBUTION IN 2-CONVEX BODIES
}

\author{
BO'AZ KLARTAG EMANUEL MILMAN
}

\begin{abstract}
We consider convex sets whose modulus of convexity is uniformly quadratic. First, we observe several interesting relations between different positions of such "2-convex" bodies; in particular, the isotropic position is a finite volume-ratio position for these bodies. Second, we prove that high dimensional 2-convex bodies posses one-dimensional marginals that are approximately Gaussian. Third, we improve for $1<p \leq 2$ some bounds on the isotropic constant of quotients of subspaces of $L_{p}$ and $S_{p}^{m}$, the Schatten Class space.
\end{abstract}

\section{INTRODUCTION}

The purpose of this note is to collect several interesting facts related to the distribution of volume in high dimensional 2-convex bodies. Suppose that $K \subset \mathbb{R}^{n}$ is a centrally-symmetric (i.e. $K=-K$ ) convex body (i.e. a convex, compact set with non-empty interior). Let $\|\cdot\|_{K}$ be the norm on $\mathbb{R}^{n}$ whose unit ball is $K$. The modulus of convexity of $K$ is the function:

$$
\delta_{K}(\varepsilon)=\inf \left\{1-\left\|\frac{x+y}{2}\right\|_{K} ;\|x\|_{K},\|y\|_{K} \leq 1,\|x-y\|_{K} \geq \varepsilon\right\},
$$

defined for $0<\varepsilon \leq 2$. We say that $K$ is "2-convex with constant $\alpha$ " (see, e.g. [LT79, Chapter 1.e]), if for all $0<\varepsilon \leq 2$,

$$
\delta_{K}(\varepsilon) \geq \alpha \varepsilon^{2} .
$$

Note that this should not be confused with the notions of $p$-convexity or q-concavity (e.g. [LT79, Chapter 1.d]) defined for Banach lattices. Being 2-convex with constant $\alpha$ is a linearly invariant property. Furthermore, as is evident from the definitions, if $K$ is 2-convex with constant $\alpha$, so is $K \cap E$ for any subspace $E$. Thus sections of a convex body inherit the 2-convexity properties of the body. The same holds

The first named author is a Clay Research Fellow and is also supported by NSF grant DMS-0456590. The second named author is supported in part by BSF and ISF. 
for projections (see, e.g. Lemma 3.4 below). A basic example of 2convex bodies are unit balls of $L_{p}$ spaces for $1<p \leq 2$, in which case $\alpha$ is of the order of $p-1$ (e.g. [LT79, Chapter 1.e]). Consequently, also sections, projections, and sections of projections of $L_{p}$-balls are 2-convex bodies, with constants that depend solely on $p$.

It is well-known that the uniform measure on a 2-convex body is "well behaved", in many senses (see, e.g. GM87] Sch95] and BL00b]). Questions on distribution of mass in high-dimensional convex sets regained some interest in the last few years, and some partial progress was obtained. We approach the study of mass distribution in 2-convex sets, in view of these developments. Arguably, the most basic question regarding volume distribution in high-dimensional convex sets is the Slicing Problem, or Hyperplane Conjecture. This question asks whether for any convex body $K \subset \mathbb{R}^{n}$ of volume one, there exists a hyperplane $H \subset \mathbb{R}^{n}$ such that $\operatorname{Vol}(K \cap H)>c$, for some universal constant $c>0$. Here and henceforth, $\operatorname{Vol}(A)$ or $|A|$ for short, denotes the volume of $A \subset \mathbb{R}^{n}$ in its affine hull. In the category of 2-convex bodies, a positive answer to this question was provided by Schmuckenschläger Sch95. We provide a more direct approach to Schmuckenschläger's result, that is based on an argument of [AdRBV98.

Proposition 1.1. Let $K \subset \mathbb{R}^{n}$ be a centrally-symmetric convex body of volume one. Suppose $K$ is 2-convex with constant $\alpha$. Then there exists a hyperplane $H \subset \mathbb{R}^{n}$ such that:

$$
\operatorname{Vol}(K \cap H) \geq c \sqrt{\alpha},
$$

where $c>0$ is a universal constant.

A centrally-symmetric convex $K \subset \mathbb{R}^{n}$ of volume one is said to be isotropic or in isotropic position, if for any $\theta \in \mathbb{R}^{n}$ :

$$
\int_{K}\langle x, \theta\rangle^{2} d x=L_{K}|\theta|^{2}
$$

where $L_{K}$ is some quantity, independent of $\theta$, and $|\cdot|$ is the Euclidean norm. In that case, the isotropic constant of $K$ is defined as $L_{K}$. It is well known (see, e.g. [MP88) that for any centrally-symmetric convex $K \subset \mathbb{R}^{n}$, there exists a linear transformation such that $\tilde{K}=T(K)$ is isotropic. Moreover, this map $T$ is unique up to orthogonal transformations. We therefore define the isotropic constant of an arbitrary centrally-symmetric convex body $K \subset \mathbb{R}^{n}$, to be $L_{K}=L_{\tilde{K}}$, where $\tilde{K}$ is an isotropic linear image of $K$. An observation that goes back to Hensley [Hen80, is that when $\mathrm{K}$ is isotropic, for any hyperplane $\mathrm{H}$ through 
the origin:

$$
\frac{c_{1}}{L_{K}} \leq \operatorname{Vol}(K \cap H) \leq \frac{c_{2}}{L_{K}}
$$

where $c_{1}, c_{2}>0$ are universal constants. Based on this, the Slicing Problem may be reformulated as follows (e.g. MP88): Is it true that for any dimension $\mathrm{n}$ and any centrally-symmetric convex body $K \subset \mathbb{R}^{n}$, we have that $L_{K} \leq C$, where $C>0$ is a universal constant?

As a by-product of our methods, we improve for $1<p \leq 2$ a bound for the isotropic constant of the unit balls of quotients of subspaces of $L_{p}$, and establish the same bound for arbitrary quotients of subspaces of $l_{p}$-Schatten-Class spaces of $m$ by $m$ matrices, denoted $S_{p}^{m}$ (see Section 3 for definitions). For a Banach Space $X$, we denote by $S Q_{n}(X)$ the family of all centrally-symmetric convex bodies $K \subset \mathbb{R}^{n}$, such that $K$ is the unit ball of some subspace of a quotient of $X$.

Proposition 1.2. Let $1<p \leq 2$, let $X=L_{p}$ or $X=S_{p}^{m}$, and suppose that $K \in S Q_{n}(X)$. Then,

$$
L_{K} \leq C \sqrt{q}
$$

where $q=p^{*}=p /(p-1)$ and $C>0$ is a universal constant.

Junge Jun94 has previously proven a version of (1.3) with $q$ in place of $\sqrt{q}$ for $X=L_{p}$. For $X=S_{p}^{m}$ and $1 \leq p \leq 2$, a universal bound on $L_{K}$ was established in KMP98, when $K$ is the unit ball of $X$, and in GP04 when $K$ is the unit ball of certain specific subspaces of $X$.

In addition to the isotropic position, there are several other important Euclidean structures that are associated with a given convex body, such as John's position, minimal mean-width position, $\ell$-position, (regular) $M$-position, etc. The relations between these various positions in general are not clear. See BKM03 for an equivalence of the hyperplane conjecture to a certain putative relation between the isotropic position and $M$-position. However, in the class of 2 -convex bodies, the following holds:

Proposition 1.3. Let $K \subset \mathbb{R}^{n}$ be a 2-convex body with constant $\alpha$ and of volume 1 . If $K$ is in isotropic position then:

$$
c \sqrt{\alpha} \sqrt{n} D_{n} \subset K
$$

where $D_{n}$ is the unit Euclidean ball in $\mathbb{R}^{n}$ and $c>0$ is a universal constant.

That is, the isotropic position of a 2-convex body is a finite volumeratio position. The volume-ratio of a centrally-symmetric convex body 
$K \subset \mathbb{R}^{n}$ is defined as:

$$
\text { v.r. }(K)=\min _{\mathcal{E} \subset K}\left(\frac{|K|}{|\mathcal{E}|}\right)^{\frac{1}{n}},
$$

where the minimum runs over all ellipsoids that are contained in $K$. If $v . r .(K)<C$, for some universal constant $C$, it is customary to say that $K$ is a finite volume-ratio body. When the minimum over all Euclidean balls is bounded by a universal constant, we will say that $K$ is in a finite volume-ratio position. Note that $c_{1}<\left|\sqrt{n} D_{n}\right|^{1 / n}<c_{2}$ for some universal constants $c_{1}, c_{2}>0$, so Proposition 1.3 implies that the isotropic position is a finite volume-ratio position.

This conclusion is clearly false for general convex bodies, even for convex bodies whose distance to the Euclidean ball is universally bounded (see the example after Lemma 2.3 below). In Section 4 we establish further rigid relations between various positions of 2-convex bodies, that cannot hold for arbitrary convex bodies. In particular, recall that $K$ is said to be in John's maximal-volume ellipsoid position when the minimum in (1.4) is attained by a Euclidean ball. We will see the following:

Proposition 1.4. Let $K \subset \mathbb{R}^{n}$ be a 2-convex body with constant $\alpha$ and of volume 1 . If $K$ is in John's maximal-volume ellipsoid position, then:

$$
\left(\int_{K}|x|^{2} d x\right)^{\frac{1}{2}} \leq \frac{C}{\alpha} \sqrt{n}
$$

where $C>0$ is a universal constant.

The latter is in a sense a converse to Proposition 1.3, since (1.5) implies that $K$ is "essentially" isotropic. To see this, note (e.g. [MP88]) that the isotropic position minimizes the value of $\int_{T(K)}|x|^{2} d x$, over all volume 1 affine images $T(K)$ of $K$, and in that case we have:

$$
\inf \left(\int_{T(K)}|x|^{2} d x\right)^{\frac{1}{2}}=\sqrt{n} L_{K} .
$$

In addition to being an "essentially" isotropic position, we show in Section 4 that John's position is in fact an "essentially" minimal meanwidth position and a 2-regular M-position (see Section 4 for definitions). A complete list of other relations between the aforementioned various positions is given at the end of Section 4 .

An additional interesting volumetric question, is the so-called "Central Limit Property of Convex Bodies". Let $X$ denote a uniformly 
distributed vector inside a convex set $K \subset \mathbb{R}^{n}$ of volume one. In its weakest form, a conjecture of Antilla, Ball and Perissinaki ABP03, states that for some non-zero vector $\theta \in \mathbb{R}^{n}$, the random variable $\langle X, \theta\rangle$ is very close to a Gaussian random variable. That is, the total variation distance between the random variable $\langle X, \theta\rangle$ and a corresponding Gaussian random variable, is smaller than $\varepsilon_{n}$, where $\varepsilon_{n}$ is a sequence tending to zero, that depends solely on $n$. In this note, we verify the following (see Theorem 5.5 for an exact formulation):

Proposition 1.5. The "Central Limit Property" holds true for arbitrary 2-convex bodies.

In ABP03, the existence of approximately Gaussian marginals of 2convex bodies was proven only under a certain, rather weak, constraint on the diameter of $K$ in isotropic position. We show in Example 4.9 that there exist 2-convex bodies in $\mathbb{R}^{n}$ for which this constraint is violated. In fact, we show that there exist such bodies of volume 1 whose diameter in isotropic position is greater than $c n$ (where $c>0$ is a universal constant). Our idea is to put $K$ in another position, namely Löwner's minimal diameter position, in which we show in Proposition 4.10 that the diameter is not larger than $\frac{C}{\lambda} n^{1-\lambda}$, where $\lambda$ depends only on $\alpha$, the 2-convexity constant of $K$ and $C>0$ is a universal constant. We conclude Proposition 1.5 by proving a version of a Theorem from ABP03] about the existence of Gaussian marginals, where the assumption of being in isotropic position is removed (see Theorem 5.3). Further developments on the existence of Gaussian marginals of uniformly convex bodies are discussed in Mil06b.

The rest of the paper is organized as follows. In Section 2 we discuss the basic volumetric properties of 2-convex bodies. In Section 3 we consider natural operations which preserve 2-convexity and its dual notion of 2-smoothness, and prove generalized versions of Proposition 1.2 Section 4 treats various positions of 2-convex bodies and their interrelations. Section 5 deals with Gaussian marginals. Throughout the text, we denote by $c, C, c^{\prime}$ etc. some positive universal constant, whose value may change from line to line. We will write $A \approx B$ to signify that $C_{1} A \leq B \leq C_{2} A$ with universal constants $C_{1}, C_{2}>0$. We denote by $D_{n}$ and $S^{n-1}$ the Euclidean unit ball and sphere in $\mathbb{R}^{n}$, respectively.

Acknowledgments. Emanuel Milman would like to sincerely thank his supervisor Prof. Gideon Schechtman for many informative discussions. 


\section{Volumetric Properties}

Let $K \subset \mathbb{R}^{n}$ be a centrally-symmetric convex body. Denote by $\|\cdot\|_{K}$ the norm whose unit ball is $K$. An equivalent well-known characterization for $K$ to be 2-convex with constant $\alpha$ (e.g. [LT79, Lemma 1.e.10]) is that for all $x, y \in \mathbb{R}^{n}$ :

$$
\|x\|_{K}^{2}+\|y\|_{K}^{2}-2\left\|\frac{x+y}{2}\right\|_{K}^{2} \geq \frac{\alpha^{\prime}}{2}\|x-y\|_{K}^{2},
$$

where the relation between $\alpha$ and $\alpha^{\prime}$ is summarized in the following:

Lemma 2.1. If $K$ is 2-convex with constant $\alpha$ then (2.1) holds with $\alpha^{\prime}=\alpha$. If (2.1) holds for all $x, y \in \mathbb{R}^{n}$, then $K$ is 2-convex with constant $\alpha=\alpha^{\prime} / 8$.

It is also known ( Nor60 ) that the Euclidean ball has the best possible modulus of convexity, implying in particular that $\alpha \leq 1 / 8$.

A basic observation due to Gromov and Milman (GM87, see also AdRBV98 for a simple proof) is that if $K$ is uniformly convex with modulus of convexity $\delta_{K}$, and $T \subset K$ with $|T| \geq \frac{1}{2}|K|$, then for any $\varepsilon>0$ :

$$
\frac{|(T+\varepsilon K) \cap K|}{|K|} \geq 1-2 e^{-2 n \delta_{K}(\varepsilon)} .
$$

We will exploit (2.2) and obtain several interesting consequences regarding mass distribution in 2-convex sets. At the heart of our argument is the following lemma, which is a direct consequence of (2.2). We prefer to give a self-contained proof, as this is a good opportunity to recreate the elegant argument from AdRBV98. This lemma was also proved in Sch95.

Lemma 2.2. Let $K \subset \mathbb{R}^{n}$ be a centrally-symmetric convex body. Assume that $K$ is 2-convex with constant $\alpha$, and that $|K|=1$. Fix $\theta \in S^{n-1}$ and denote $w=\sup _{x \in K}|\langle x, \theta\rangle|$. Then for any $t>0$ :

$$
\operatorname{Vol}\{x \in K ;\langle x, \theta\rangle>t\} \leq 2 \exp \left(-2 \alpha n(t / w)^{2}\right) .
$$

Proof. Let $A(t)=\{x \in K ;\langle x, \theta\rangle>t\}$ and put $B=\{x \in K ;\langle x, \theta\rangle<$ $0\}$. Note that if $x \in A(t), y \in B$ then $\|x-y\|_{K} \geq \frac{t}{w}$. According to the definition of 2-convexity,

$$
\frac{B+A(t)}{2} \subset\left(1-\alpha\left(\frac{t}{w}\right)^{2}\right) K .
$$


By the Brunn-Minkowski inequality,

$$
\sqrt{|B| \cdot|A(t)|} \leq\left|\frac{B+A(t)}{2}\right| \leq\left(1-\alpha(t / w)^{2}\right)^{n} \leq \exp \left(-\alpha n(t / w)^{2}\right) .
$$

Since $|B|=1 / 2$, we have:

$$
|A(t)| \leq 2 \exp \left(-2 \alpha n(t / w)^{2}\right) .
$$

Next, we present several consequences of Lemma 2.2. The first one is the following observation.

Lemma 2.3. Let $K \subset \mathbb{R}^{n}$ be a centrally-symmetric convex body. Assume that $K$ is 2-convex with constant $\alpha$ and volume 1 , and that $K$ is isotropic. Then:

$$
c \sqrt{\alpha} \sqrt{n} L_{K} D_{n} \subset K
$$

where $c>0$ is a universal constant.

Proof. Let $\theta \in S^{n-1}$ be arbitrary. For $t \in \mathbb{R}$ set

$$
A(t)=K \cap\left\{x \in \mathbb{R}^{n} ;\langle x, \theta\rangle<t\right\},
$$

and denote $f(t)=|A(t)|$. As before, we use $w=\sup _{x \in K}|\langle x, \theta\rangle|$ to denote the width of $K$ in direction $\theta$. By Lemma 2.2. we see that for $t>0$ :

$$
f(t) \geq 1-2 \exp \left(-2 \alpha n(t / w)^{2}\right) .
$$

On the other-hand, $f^{\prime}(t)=|K \cap\{\langle x, \theta\rangle=t\}|$ is a log-concave function by Brunn-Minkowski which is even, and therefore attains its maximum at 0 . Since $f^{\prime}(0) \approx 1 / L_{K}$ (e.g. [MP88]), we see that:

$$
f(t) \leq f(0)+t f^{\prime}(0) \leq \frac{1}{2}+c \frac{t}{L_{K}}
$$

Choosing $t=L_{K} / 4 c$ and combining (2.3) and (2.4), we see that $w \geq$ $c^{\prime} \sqrt{\alpha} \sqrt{n} L_{K}$. Since the direction $\theta \in S^{n-1}$ was arbitrary, the lemma follows.

Lemma 2.3 entails Proposition 1.1 and Proposition 1.3 at once. Indeed, since $\left|\sqrt{n} D_{n}\right|^{1 / n} \approx 1$ and $|K|=1$, Lemma 2.3 implies that $L_{K} \leq c / \sqrt{\alpha}$. Proposition [1.1 immediately follows (see, e.g. [MP88]). Since also $c<L_{K}$ (e.g. MP88]), then Lemma 2.3 implies that:

$$
c \sqrt{\alpha} \sqrt{n} D \subset K
$$

and Proposition 1.3 is established. Note that it is quite unusual for a convex body to contain a large Euclidean ball in isotropic position, even when the body has a small volume-ratio. For instance, consider 
the convex body $K=\left\{x \in \mathbb{R}^{n} ;|x| \leq \sqrt{n},\left|x_{1}\right| \leq 1\right\}$, and let $\tilde{K}$ be an isotropic linear image of $K$. It is easily seen that $\tilde{K}$ does not contain a ball of radius larger than $c$, although $K$ is isomrophic to a Euclidean ball, and clearly has a finite volume-ratio.

Another consequence of Lemma 2.2 it the following Proposition. As usual, the dual norm to $\|\cdot\|_{K}$ is defined by $\|x\|_{K}^{*}=\sup _{y \in K}\langle x, y\rangle$, and its unit ball is called the polar body to $K$, and denoted $K^{\circ}$. For $\theta \in S^{n-1}$, we define the $\psi_{2}$-norm of the linear functional $\langle\cdot, \theta\rangle$ w.r.t. the uniform measure on $K$ as:

$$
\|\langle\cdot, \theta\rangle\|_{L_{\psi_{2}(K)}}:=\inf \left\{\lambda>0 ; \frac{1}{|K|} \int_{K} e^{\frac{\langle x, \theta\rangle^{2}}{\lambda^{2}}} d x \leq 2\right\} .
$$

The $L_{p}$-norm is defined as:

$$
\|\langle\cdot, \theta\rangle\|_{L_{p}(K)}:=\left(\frac{1}{|K|} \int_{K}|\langle x, \theta\rangle|^{p} d x\right)^{1 / p} .
$$

It is well-known (e.g. [JSZ85, Proposition 3.6]) that:

$$
\|\langle\cdot, \theta\rangle\|_{L_{\psi_{2}(K)}} \approx \sup _{p \geq 2} \frac{\|\langle\cdot, \theta\rangle\|_{L_{p}(K)}}{\sqrt{p}},
$$

implying in particular that:

$$
\|\langle\cdot, \theta\rangle\|_{L_{\psi_{2}(K)}} \geq C \frac{\|\theta\|_{K}^{*}}{\sqrt{n}}
$$

since $\|\theta\|_{K}^{*} \approx\|\langle\cdot, \theta\rangle\|_{L_{n}(K)}$ (e.g. [Pao02]). Lemma 2.2 therefore implies:

Proposition 2.4. Let $K \subset \mathbb{R}^{n}$ be a centrally-symmetric 2-convex body with constant $\alpha$. Then for all $\theta \in S^{n-1}$ :

$$
C_{1} \frac{\|\theta\|_{K}^{*}}{\sqrt{n}} \leq\|\langle\cdot, \theta\rangle\|_{L_{\psi_{2}(K)}} \leq C_{2} \frac{\|\theta\|_{K}^{*}}{\sqrt{\alpha} \sqrt{n}},
$$

where $C_{1}, C_{2}>0$ are two universal constants.

Proposition 2.4 provides us with a way to find directions $\theta \in S^{n-1}$ for which $\operatorname{Vol}\{x \in K ;\langle x, \theta\rangle \geq t\}$ decays in a sub-gaussian rate, as reflected by $\|\langle\cdot, \theta\rangle\|_{L_{\psi_{2}(K)}}$. As a first application, note that for any convex body of volume one, there exists a direction in which the width is smaller than $C \sqrt{n}$ (otherwise the body would contain a Euclidean ball of volume greater than one). Together with a straightforward application of Markov's inequality, and denoting $M^{*}(K)=\int_{S^{n-1}}\|\theta\|^{*} d \sigma(\theta)$, we conclude the following immediate corollary of Proposition 2.4 
Corollary 2.5. Let $K \subset \mathbb{R}^{n}$ be a centrally-symmetric convex body. Assume that $K$ is 2-convex with constant $\alpha$ and volume 1 . Then there exists a universal constant $C>0$ such that:

(1) There exists $a \theta \in S^{n-1}$ such that:

$$
\|\langle\cdot, \theta\rangle\|_{L_{\psi_{2}(K)}} \leq C / \sqrt{\alpha} \text {. }
$$

$$
\sigma\left\{\theta \in S^{n-1} ;\|\langle\cdot, \theta\rangle\|_{L_{\psi_{2}}(K)} \leq C \frac{M^{*}(K)}{\sqrt{\alpha} \sqrt{n}}\right\} \geq \frac{1}{2}
$$

In Section 4, we will see several positions of a 2-convex body $K$ of volume 1 for which $M^{*}(K) \leq C \sqrt{n}$. The last corollary implies that in these positions, at least half of the directions have $\psi_{2}$-decay. We say that a body satisfying:

$$
\|\langle\cdot, \theta\rangle\|_{L_{\psi_{2}}(K)} \leq A \cdot|K|^{1 / n}
$$

for all $\theta \in S^{n-1}$ is a $\psi_{2}$ body (with constant $A$ ). In general, a 2-convex body is not a $\psi_{2}$ body. Indeed, as apparent from (2.5), a $\psi_{2}$ body (with constant $A$ ) of volume 1 always satisfies $\operatorname{diam}(K) \leq C A \sqrt{n}$, but any $l_{p}^{n}$ for $p<2$ (normalized to have volume 1 ) already fails to satisfy this (with a universal constant $A$ ) for large enough $n$. Here and henceforth, $\operatorname{diam}(K)$ denotes the diameter of $K$. Nevertheless, we can still say the following:

Proposition 2.6. Let $K \subset \mathbb{R}^{n}$ be a centrally-symmetric convex body. Assume that $K$ is 2 -convex with constant $\alpha$, has volume 1 and that it is isotropic. Then a random $\lfloor n / 2\rfloor$ dimensional section of $K$ is a $\psi_{2}$-body with high probability.

Proof. By definition, any section of $K$ is a 2-convex body with the same constant. By Proposition 1.3, the isotropic position is also a finite volume-ratio position for $K$, and $c \sqrt{\alpha} \sqrt{n} D_{n} \subset K$. But by a classical result of [Sza80] and [STJ80] (based on [Kaš77]), a random $\lfloor n / 2\rfloor$ dimensional section $L \cap E$ of a convex body $L$ containing $D_{n}$ is isomorphic to a Euclidean ball, and in particular satisfies $\operatorname{diam}(L \cap E) \leq C\left(|L| /\left|D_{n}\right|\right)^{2 / n}$ with probability greater than $1-(1 / 2)^{n}$. Therefore:

$$
c \sqrt{\alpha} \sqrt{n}\left(D_{n} \cap E\right) \subset K \cap E \subset \frac{C^{\prime}}{\sqrt{\alpha}} \sqrt{n}\left(D_{n} \cap E\right)
$$

with the same probability. Applying Proposition 2.4 to $K \cap E$ and using the left-hand-side of (2.6) to compensate for the volume of $K \cap E$, we see that:

$$
\|\langle\cdot, \theta\rangle\|_{L_{\psi_{2}(K \cap E)}} \leq \frac{C^{\prime}}{\alpha^{3 / 2}}|K \cap E|^{2 / n}
$$


for all $\theta \in S^{n-1} \cap E$. This concludes the proof.

\section{OperATIONS PRESERVING 2-CONVEXITY}

We have already seen that (by definition) any section of a 2-convex body with constant $\alpha$ is itself a 2-convex body with the same constant. In this section we will consider several additional natural operations which preserve 2-convexity and the dual notion of 2-smoothness, and conclude with several new results on the isotropic constant of different families of bodies.

The first natural operation to consider is taking projections. Since this is the dual operation to taking sections, it will be convenient to first introduce the dual notion to 2-convexity, which is 2-smoothness. The modulus of smoothness of $K$ is defined as the following function for $\tau>0$ :

$$
\rho_{K}(\tau)=\sup \left\{\frac{\|x+y\|_{K}+\|x-y\|_{K}}{2}-1 ;\|x\|_{K} \leq 1,\|y\|_{K} \leq \tau\right\} .
$$

A body $K$ is called "2-smooth with constant $\beta$ " (see, e.g. [LT79, Chapter 1.e]), if for all $\tau>0$ :

$$
\rho_{K}(\tau) \leq \beta \tau^{2}
$$

It is well-known (e.g. [LT79]) that the modulus of smoothness is dual to the modulus of convexity (this can be carefully formalized using Legendre transforms). We summarize Propositions 1.e.2 and 1.e.6 from [LT7] in the following:

Lemma 3.1. Let $K$ be a centrally-symmetric convex body in $\mathbb{R}^{n}$. Then $K$ is 2-convex with constant $\alpha$ iff $K^{\circ}$ is 2 -smooth with constant $\frac{1}{16 \alpha}$.

We will frequently refer to the Blaschke-Santalo inequality (San49, the r.h.s. below) and its reverse form due to Bourgain-Milman ([BM87], the l.h.s. below), which together state that for any convex body $K$ :

$$
c \leq\left(\frac{|K|}{\left|D_{n}\right|}\right)^{1 / n}\left(\frac{\left|K^{\circ}\right|}{\left|D_{n}\right|}\right)^{1 / n} \leq 1
$$

Lemma 3.1. coupled with the Blaschke-Santalo inequality or its reverse form, imply that we can translate many volumetric results on 2-convex bodies to 2 -smooth bodies. In particular, Proposition 1.3 translates to the fact that 2 -smooth bodies have finite outer-volumeratio. We define the outer-volume-ratio of a body $K$ as:

$$
\text { o.v.r. }(K)=\inf _{\mathcal{E} \supset K}\left(\frac{|\mathcal{E}|}{|K|}\right)^{\frac{1}{n}}
$$


where the infimum runs over all ellipsoids that contain $K$. If o.v.r. $(K)<$ $C$, for some universal constant $C>0$, it is customary to say that $K$ has finite outer-volume-ratio. It is well known (e.g. MP88) that $L_{K} \leq C^{\prime}$ o.v.r. $(K)$ for any convex body $K$. Combining everything together, we have the following useful:

Proposition 3.2. Let $K$ be a 2-smooth convex body with constant $\beta$. Then o.v.r. $(K) \leq C \sqrt{\beta}$. In particular, $L_{K} \leq C^{\prime} \sqrt{\beta}$.

Note that if $K \subset T$ then o.v.r. $(K) \leq(|T| /|K|)^{1 / n}$ o.v.r. $(T)$. The following is therefore an immediate corollary of Proposition 3.2 .

Corollary 3.3. Let $K$ be a centrally-symmetric convex body in $\mathbb{R}^{n}$. Then:

$$
L_{K} \leq C \inf \left\{\sqrt{\beta}\left(\frac{|T|}{|K|}\right)^{1 / n} \mid \begin{array}{c}
K \subset T, \\
T \text { is 2-smooth with constant } \beta
\end{array}\right\}
$$

We can now turn to investigate the action of taking projections of 2-convex and 2-smooth bodies. For a subspace $E \subset \mathbb{R}^{n}$, we denote by $\operatorname{Proj}_{E}$ the orthogonal projection onto $E$. As evident from the definitions, any section of a 2 -smooth body with constant $\beta$ is itself a 2 -smooth body with the same constant. By passing to the polar body and using Lemma 3.1, the duality between sections and projections immediately implies:

Lemma 3.4. Let $K \subset \mathbb{R}^{n}$ be a 2-convex (2-smooth) body with constant $\gamma$. Then so is $\operatorname{Proj}_{E}(K)$, with the same constant $\gamma$, for any subspace $E \subset \mathbb{R}^{n}$.

Using Lemma 3.4, a remarkable consequence of Proposition 2.4 is that the $\psi_{2}$-norm of the linear functional $\langle\cdot, x\rangle$ on a projection $\operatorname{Proj}_{E}(K)$ of a 2-convex body $K$, essentially depends (up to universal constants) only on $x \in E$ and not on the subspace $E$. More precisely:

Proposition 3.5. Let $K \subset \mathbb{R}^{n}$ be a 2-convex body with constant $\alpha$, and let $E$ be a $k$-dimensional subspace. Then for any $x \in E$ :

$$
C_{1}\|x\|_{K}^{*} \leq\|\langle\cdot, x\rangle\|_{L_{\psi_{2}\left(\operatorname{Proj}_{E}(K)\right)}} \sqrt{k} \leq C_{2} \frac{1}{\sqrt{\alpha}}\|x\|_{K}^{*}
$$

This is one of the rare cases where we can deduce volumetric information on $\operatorname{Proj}_{E}(K)$ from that of $K$. Typically, these two bodies have different volumetric behaviour.

Let us consider other natural operations which preserve 2-convexity. Unfortunately, the Minkowski sum is a bad candidate for this. Indeed, 
even in $\mathbb{R}^{2}$, the sum of two very narrow ellipsoids which are perpendicular to each other, may be brought arbitrarily close to a square, which is not 2-uniformly convex. Nevertheless, there exists a well known natural summation operation, which actually preserves both 2-uniform convexity and 2-uniform smoothness. Recall that the 2-Firey sum of two convex bodies $K$ and $T$, denoted by $K+{ }_{2} T$, is defined as the unit ball of the norm satisfying:

$$
\|z\|_{K+{ }_{2} T}^{2}=\inf _{z=x+y}\|x\|_{K}^{2}+\|y\|_{T}^{2} .
$$

It is easy to see that the dual norms satisfy:

$$
\left(\|z\|_{K+_{2} T}^{*}\right)^{2}=\left(\|z\|_{K}^{*}\right)^{2}+\left(\|z\|_{T}^{*}\right)^{2}
$$

We will refer to the latter operation as 2-Firey intersection, and denote the 2-Firey intersection of $K$ and $T$ as $K \cap_{2} T$. Note that $\left(K \cap_{2} T\right)^{\circ}=$ $K^{\circ}+{ }_{2} T^{\circ}$.

Lemma 3.6. Let $K$ and $T$ be 2-convex (smooth) bodies with constants $\gamma_{K}$ and $\gamma_{T}$, respectively. Then so is their 2-Firey sum $K+_{2} T$ and intersection $K \cap_{2} T$, with constant $\min \left\{\gamma_{K}, \gamma_{T}\right\} / 8\left(\max \left\{\gamma_{K}, \gamma_{T}\right\} \cdot 8\right)$.

Proof. Obviously there is no loss in generality in assuming that $\gamma_{K}=$ $\gamma_{T}=\gamma$. Since $\left(K \cap_{2} T\right)^{\circ}=K^{\circ}+{ }_{2} T^{\circ}$, Lemma 3.1 implies that the case of 2 -smooth bodies follows from the case of 2 -convex bodies by duality. We will therefore restrict ourselves to the latter case, and assume that $K$ and $T$ are 2-convex with constant $\gamma$.

By Lemma 2.1, we have for $G=K, T$ and for all $x, y \in \mathbb{R}^{n}$ :

$$
\|x\|_{G}^{2}+\|y\|_{G}^{2}-2\left\|\frac{x+y}{2}\right\|_{G}^{2} \geq \frac{\gamma}{2}\|x-y\|_{G}^{2} .
$$

Summing these two inequalities for $G=K$ and $G=T$, we see that (3.3) is also satisfied for $G=K \cap_{2} T$. Using Lemma 2.1 again, this implies that $K \cap_{2} T$ is 2-convex with constant $\gamma / 8$.

Next, for any $z_{1}, z_{2} \in \mathbb{R}^{n}$, write $z_{i}=x_{i}^{K}+x_{i}^{T}$ so that:

$$
\left\|z_{i}\right\|_{K+{ }_{2} T}^{2}=\left\|x_{i}^{K}\right\|_{K}^{2}+\left\|x_{i}^{T}\right\|_{T}^{2}
$$

(by compactness the infimum is achieved). By Lemma 2.1] we know that for $G=K, T$ :

$$
\left\|x_{1}^{G}\right\|_{G}^{2}+\left\|x_{2}^{G}\right\|_{G}^{2} \geq 2\left\|\frac{x_{1}^{G}+x_{2}^{G}}{2}\right\|_{G}^{2}+\frac{\gamma}{2}\left\|x_{1}^{G}-x_{2}^{G}\right\|_{G}^{2} .
$$


Summing these two inequalities for $G=K$ and $G=T$ and denoting $Z=K+{ }_{2} T$, we have:

$$
\begin{aligned}
& \left\|z_{1}\right\|_{Z}^{2}+\left\|z_{2}\right\|_{Z}^{2}=\left\|x_{1}^{K}\right\|_{K}^{2}+\left\|x_{2}^{K}\right\|_{K}^{2}+\left\|x_{1}^{T}\right\|_{T}^{2}+\left\|x_{2}^{T}\right\|_{T}^{2} \\
\geq & 2\left(\left\|\frac{x_{1}^{K}+x_{2}^{K}}{2}\right\|_{K}^{2}+\left\|\frac{x_{1}^{T}+x_{2}^{T}}{2}\right\|_{T}^{2}\right)+\frac{\gamma}{2}\left(\left\|x_{1}^{K}-x_{2}^{K}\right\|_{K}^{2}+\left\|x_{1}^{T}-x_{2}^{T}\right\|_{T}^{2}\right) \\
\geq & 2\left\|\frac{z_{1}+z_{2}}{2}\right\|_{Z}^{2}+\frac{\gamma}{2}\left\|z_{1}-z_{2}\right\|_{Z}^{2},
\end{aligned}
$$

where the last inequality follows from the definition of $Z=K+{ }_{2} T$ and the fact that $z_{1}+z_{2}=\left(x_{1}^{K}+x_{2}^{K}\right)+\left(x_{1}^{T}+x_{2}^{T}\right)$ and $z_{1}-z_{2}=$ $\left(x_{1}^{K}-x_{2}^{K}\right)+\left(x_{1}^{T}-x_{2}^{T}\right)$. Lemma 2.1 implies that $K+{ }_{2} T$ is 2-convex with constant $\gamma / 8$.

Remark 3.7. It is important to emphasize that the additional factor of 8 appearing in the Lemma is immaterial, and that the Lemma holds in full generality when summing (intersecting) an arbitrary number of bodies (with the same constant factor of 8).

We can now summarize our bounds for the isotropic constant in the following statements. For a Banach space $X$, we denote by $S Q_{n}(X)$ the class of unit balls of $n$-dimensional subspaces of quotients of $X$. We denote $F_{2}^{0} S Q_{n}(X)=S Q_{n}(X)$, and by induction:

$$
F_{2}^{i+1} S Q_{n}(X)=\left\{\bigwedge_{i=1}^{l} \bigoplus_{j=1}^{m_{i}} K_{j}^{i} ;\left\{K_{j}^{i}\right\} \subset F_{2}^{i} S Q_{n}(X)\right\}
$$

where $\bigwedge$ and $\bigoplus$ denote 2-Firey intersection and sum, respectively. We set $F_{2} S Q_{n}(X)=\cup_{i=0}^{\infty} F_{2}^{i} S Q_{n}(X)$. Note that it is possible to make the class $F_{2} S Q_{n}(X)$ even richer, by alternately taking subspaces, quotients, 2-Firey sums and 2-Firey intersections (since the operation of 2-Firey sum is not distributive w.r.t. taking subspace or 2-Firey intersection) starting from $X$, but this is a complication which we wish to avoid. Lemmas 3.4 and 3.6. together with Remark 3.7, show that if $X$ is 2convex (2-smooth) with constant $\alpha(\beta)$, then so is every member of $F_{2} S Q_{n}(X)$ with constant $\alpha / 8(8 \beta)$. Corollary 3.3 therefore implies:

Theorem 3.8. Let $K$ be a centrally-symmetric convex body in $\mathbb{R}^{n}$, and let $X$ be a 2-smooth Banach space with constant $\beta$. Then:

$$
L_{K} \leq C \sqrt{\beta} \inf \left\{\left(\frac{|T|}{|K|}\right)^{1 / n} \mid K \subset T, T \in F_{2} S Q_{n}(X)\right\} .
$$


Consider $X=L_{p}$ for $2 \leq p<\infty$ in Theorem 3.8. Note that $X^{*}=L_{q}$ with $q=1+1 /(p-1)$, for which it is known (e.g. [LT79, p. 63]) that $X^{*}$ is 2 -convex with constant equivalent to $1 /(p-1)$. By Lemma 3.1 this implies that $X$ is 2 -smooth with constant bounded by $C(p-1)$. We therefore have:

Corollary 3.9. Let $K$ be a centrally-symmetric convex body in $\mathbb{R}^{n}$. Then:

$$
L_{K} \leq C \inf \left\{\sqrt{p}\left(\frac{|T|}{|K|}\right)^{1 / n} \mid K \subset T, T \in F_{2} S Q_{n}\left(L_{p}\right), p \geq 2\right\} .
$$

This is a generalization of one half (the range $p \geq 2$ ) of a Theorem of Junge (Jun94, see also [Mil06a]):

\section{Theorem (Junge).}

$$
L_{K} \leq C \inf \left\{\sqrt{p} q\left(\frac{|T|}{|K|}\right)^{1 / n} \mid \begin{array}{c}
K \subset T, T \in S Q_{n}\left(L_{p}\right), \\
1<p<\infty, 1 / p+1 / q=1
\end{array}\right\} .
$$

In fact, Junge showed that $L_{p}$ may be replaced by any Banach space $X$ with finite type and bounded $g l_{2}(X)$ (the Gordon-Lewis constant of $X$ ), in which case $\sqrt{p} q$ above should be replaced by some constant depending on $X$.

We can also improve the second half of Junge's Theorem (in the range $1<p \leq 2$ ) by replacing the factor of $q$ by $\sqrt{q}$. Unfortunately, with our approach we have to insist that $K$ itself is in $F_{2} S Q_{n}\left(L_{p}\right)$. Our version reads as follows:

Theorem 3.10. Let $K \in F_{2} S Q_{n}\left(L_{p}\right)$ for $1<p \leq 2$, and let $q$ be given by $1 / p+1 / q=1$. Then:

$$
L_{K} \leq C \sqrt{q}
$$

The latter is an immediate corollary of the the fact that $L_{p}$ for $1<p \leq 2$ is 2 -convex with constant equivalent to $p-1$ (e.g. LT79, Chapter 1.e]), combined with the following general Theorem, which is a consequence of Proposition 1.1.

Theorem 3.11. Let $X$ be a 2-convex Banach space with constant $\alpha$, and let $K \in F_{2} S Q_{n}(X)$. Then:

$$
L_{K} \leq C \frac{1}{\sqrt{\alpha}}
$$

Another interesting example is obtained by taking $X$ to be the space of all $m$ by $m$ complex or real matrices, equipped with the norm $\|A\|=$ $\left(\operatorname{tr}\left(A A^{*}\right)^{p / 2}\right)^{1 / p}$, the so-called $l_{p^{-}}$Schatten-Class which will be denoted 
by $S_{p}^{m}$. It was observed in KMP98, that the isotropic constants of these spaces are uniformly bounded (in $m$ ), which is especially interesting in the range $1 \leq p<2$, since for $p \geq 2$ it is known that the unit ball of $S_{p}^{m}$ (or any of its subspaces) has finite outer volume-ratio. In the former range, it has been recently shown in GP04 that (in particular) the isotropic constants of several special subspaces of $S_{p}^{m}$ are also uniformly bounded. Although our method does not extend to $p=1$, we can show the following result, which in particular demonstrates that the same is true for any subspace of quotient of $S_{p}^{m}$, provided that $p$ is bounded away from 1. The modulus of convexity (and smoothness) of $S_{p}^{m}$ was estimated by N. Tomczak-Jaegermann in [TJ74], where it was shown that $\delta_{S_{p}^{m}} \approx \delta_{L_{p}}$. It follows that $S_{p}^{m}$ is 2-convex with constant equivalent to $p-1$ for $1<p \leq 2$, which together with Theorem 3.11 gives:

Theorem 3.12. Let $K \in F_{2} S Q_{n}\left(S_{p}^{m}\right)$ for $1<p \leq 2$ and $m \geq n$, and let $q$ be given by $1 / p+1 / q=1$. Then:

$$
L_{K} \leq C \sqrt{q}
$$

It is clear that the case $p=1$ in Theorem 3.10 and Theorem 3.12 must serve as a break-down point for our method. Indeed, since $S_{1}^{m}$ contains $l_{1}^{m}$ as a subspace (of the diagonal matrices), and since every convex body may be approximated as the unit ball of a quotient of $l_{1}^{m}$ for large-enough $m$, or simply as the quotient of $L_{1}$, a similar result for $p=1$ in either theorem would solve the Slicing Problem.

\section{Equivalence Between positions of 2-CONVEX Bodies}

For the results of this section, we will need to recall a few basic notions from Banach space theory. The (Rademacher) type- $p$ constant of a Banach space $X$ (for $1 \leq p \leq 2$ ), denoted $T_{p}(X)$, is the minimal $T>0$ for which:

$$
\left(\mathbb{E}\left\|\sum_{i=1}^{m} \varepsilon_{i} x_{i}\right\|^{2}\right)^{1 / 2} \leq T\left(\sum_{i=1}^{m}\left\|x_{i}\right\|^{2}\right)^{1 / 2}
$$

for any $m \geq 1$ and any $x_{1}, \ldots, x_{m} \in X$, where $\left\{\varepsilon_{i}\right\}$ are independent, identically distributed random variables uniformly distributed on $\{-1,1\}$ and $\mathbb{E}$ denotes expectation. Similarly, the cotype- $q$ constant of $X$ (for $2 \leq q \leq \infty$ ), denoted $C_{q}(X)$, is the minimal $C>0$ for which:

$$
\left(\mathbb{E}\left\|\sum_{i=1}^{m} \varepsilon_{i} x_{i}\right\|^{2}\right)^{1 / 2} \geq \frac{1}{C}\left(\sum_{i=1}^{m}\left\|x_{i}\right\|^{q}\right)^{1 / q}
$$


for any $m \geq 1$ and $x_{1}, \ldots, x_{m} \in X$. We say that $X$ has type $p$ (cotype $q)$ if $T_{p}(X)<\infty\left(C_{q}(X)<\infty\right)$. We also say that $X$ is of type $p$ (cotype $q)$ if $p=\sup \left\{p^{\prime} ; X\right.$ has type $\left.p^{\prime}\right\}\left(q=\inf \left\{q^{\prime} ; X\right.\right.$ has cotype $\left.\left.q^{\prime}\right\}\right)$.

Let $L_{2}\left(\{-1,1\}^{m}, X\right)$ denote the space of $X$-valued functions on the discrete cube $\{-1,1\}^{m}$, equipped with the norm $\left(\mathbb{E}\left\|f\left(\varepsilon_{1}, \ldots, \varepsilon_{m}\right)\right\|^{2}\right)^{1 / 2}$. We denote by $\operatorname{Rad}_{m}(X)$ the Rademacher projection on $L_{2}\left(\{-1,1\}^{m}, X\right)$ (see [MS86]), and denote $\|\operatorname{Rad}(X)\|=\sup _{m}\left\|\operatorname{Rad}_{m}(X)\right\|$ where $\left\|\operatorname{Rad}_{m}(X)\right\|$ is the operator norm of $\operatorname{Rad}_{m}(X)$. By duality, it is easy to verify that $\left\|\operatorname{Rad}\left(X^{*}\right)\right\|=\|\operatorname{Rad}(X)\|$, and it is clear that $\left\|\operatorname{Rad}_{m}(X)\right\|=$ $\sup _{E \subset X}\left\|\operatorname{Rad}_{m}(E)\right\|$ where the supremum runs over all finite-dimensional subspaces of $X$.

One of the most important results in the so-called local-theory of Banach spaces is a theorem by Pisier who showed that $\|\operatorname{Rad}(X)\|$ may be bounded from above by an (explicit) function of $T_{p}(X)$ when $p>1$, concluding that $\|\operatorname{Rad}(X)\|<\infty$ when $X$ has type $p>1$. When $p=2$, there is a much easier argument, going back to a remark at the end of the work by Maurey and Pisier [MP76] (see also BTV00, Remark 2.11] for an explicit proof), showing (without any constants!):

Lemma 4.1. $\|\operatorname{Rad}(X)\| \leq T_{2}(X)$.

The next lemma, which gives a non-quantitive estimate of the opposite inequality (for the general $p$ case) using a compactness argument, is a known consequence of the Maurey-Pisier Theorem [MP76]:

Lemma 4.2. There exists a function $C(R): \mathbb{R}_{+} \rightarrow \mathbb{R}_{+}$such that any finite-dimensional Banach space $X$ with $\|\operatorname{Rad}(X)\| \leq R$ satisfies $T_{p(R)}(X) \leq C(R)$ with $p(R)=1+1 / C(R)$.

Sketch of proof. Assume that this is not true for some $R>0$. This means that there exist finite-dimensional Banach spaces $X_{i}$ with $\left\|\operatorname{Rad}\left(X_{i}\right)\right\| \leq$ $R$ and $T_{1+1 / i}\left(X_{i}\right)>i$. The latter easily implies that $\operatorname{dim}\left(X_{i}\right) \rightarrow \infty$, since always $T_{p}\left(X_{i}\right) \leq T_{2}\left(X_{i}\right) \leq \sqrt{\operatorname{dim}\left(X_{i}\right)}$ for any $1 \leq p \leq 2\left(X_{i}\right.$ is $\sqrt{\operatorname{dim}\left(X_{i}\right)}$-isomorphic to a Hilbert space $H_{i}$ by John's Theorem, and $\left.T_{2}\left(H_{i}\right)=1\right)$. We now construct an infinite dimensional Banach space $X$ as the $l_{2}$ sum of the $X_{i}$ 's, i.e. for $x=\left(x_{i}\right)_{i \geq 1}$ with $x_{i} \in X_{i}$ define $\|x\|_{X}=\left(\sum_{i>1}\left\|x_{i}\right\|_{X_{i}}^{2}\right)^{\frac{1}{2}}$ and set $X=\left\{x ;\|x\|_{X}<\infty\right\}$ endowed with the norm $\|\cdot\|_{X}$. It is elementary to check that $\|\operatorname{Rad}(X)\| \leq R$, and since $X$ contains each $X_{i}$ as a subspace we must have that $X$ is of type 1 . The latter implies by the Maurey-Pisier Theorem (actually we only need the type 1 case, which is due to Pisier [Pis73] that $X$ contains $(1+\epsilon)$ isometric copies of $l_{1}^{m}$ for arbitrary $\epsilon>0$ and $m$, and as a consequence $\|\operatorname{Rad}(X)\| \geq \sup _{m}\left\|\operatorname{Rad}\left(l_{1}^{m}\right)\right\|=\infty$. We arrive to a contradiction, so the assertion is proved. 
Let us return to the study of 2 -convex bodies. We recall the following classical result (e.g. [LT79, Theorem 1.e.16]). For completeness, we sketch the proof.

\section{Lemma 4.3.}

(1) Let $K$ be a 2-convex body with constant $\alpha$. Then $C_{2}\left(X_{K}\right) \leq \frac{C}{\sqrt{\alpha}}$.

(2) Let $K$ be a 2-smooth body with constant $\beta$. Then $T_{2}\left(X_{K}\right) \leq$ $C \sqrt{\beta}$.

Proof. (1) easily follows from the equivalent characterization (2.1) of a 2-convex body, which asserts that for any $x_{1}, x_{2} \in \mathbb{R}^{n}$ :

$$
\mathbb{E}\left\|\varepsilon_{1} x_{1}+x_{2}\right\|^{2}=\frac{1}{2}\left(\left\|x_{2}+x_{1}\right\|^{2}+\left\|x_{2}-x_{1}\right\|^{2}\right) \geq \alpha\left\|x_{1}\right\|^{2}+\left\|x_{2}\right\|^{2} .
$$

Hence by induction, since $\alpha<1$ :

$$
\mathbb{E}\left\|\sum_{i=1}^{m} \varepsilon_{i} x_{i}\right\|^{2} \geq \alpha \sum_{i=1}^{m}\left\|x_{i}\right\|^{2},
$$

for any $x_{1}, \ldots, x_{m} \in \mathbb{R}^{m}$, which concludes the proof of (1) (even without a constant!). (2) follows either by duality or similarly from the equivalent characterization of a 2-smooth body (e.g. [BL00a, Theorem A.7]):

$$
\|x+y\|^{2}+\|x-y\|^{2}-2\|x\|^{2} \leq C \beta\|y\|^{2},
$$

for every $x, y \in \mathbb{R}^{n}$.

We are now ready to conclude the following useful:

Lemma 4.4. Let $K$ be a 2-convex body with constant $\alpha$. Then:

$$
\left\|\operatorname{Rad}\left(X_{K}\right)\right\| \leq C / \sqrt{\alpha} .
$$

(2) There exists a $p>1$ which depends on $\alpha$ only, such that:

$$
T_{p}\left(X_{K}\right) \leq 1 /(p-1)
$$

Proof. By Lemma 3.1, $K^{\circ}$ is 2 -smooth with constant $1 /(16 \alpha)$, and so by Lemmas 4.1 and 4.3 we see that:

$$
\|\operatorname{Rad}(X)\|=\left\|\operatorname{Rad}\left(X^{*}\right)\right\| \leq T_{2}\left(X^{*}\right) \leq \frac{C}{\sqrt{\alpha}}
$$

which concludes the proof of (1). Applying Lemma 4.2. we immediately deduce (2). 
Lemmas 4.3 and 4.4 allow us to deduce several interesting results about 2-convex bodies. By a classical result of Figiel and TomzcakJaegermann on the $l$-position ([FTJ79]), for any convex body $K$ there exists a position for which $M(K) M^{*}(K) \leq C\left\|\operatorname{Rad}\left(X_{K}\right)\right\|$, and in fact this is satisfied in the minimal mean-width position. The latter is defined (up to orthogonal rotations) as the volume-preserving affine image of $K$ for which $M^{*}(K)$ is minimal. Recall that we always have:

$$
\frac{1}{M(K)} \leq \text { Vol.rad. }(K) \leq M^{*}(K)
$$

where Vol.rad. $(K)=\left(|K| /\left|D_{n}\right|\right)^{1 / n}$ and the first inequality follows from Jensen's inequality while the second is Urysohn's inequality. We therefore deduce that in the minimal mean-width position, a 2-convex body $K$ with constant $\alpha$ satisfies:

$$
M^{*}(K) \leq \frac{C}{\sqrt{\alpha}} \operatorname{Vol} . r a d .(K),
$$

which is essentially the best possible by (4.1). We will refer to (4.2) as " $M^{*}(K)$ is bounded", omitting the reference to the volume-radius. As we shall see, there are many advantages of working with a position in which $M^{*}(K)$ is bounded.

Our next Proposition shows that whenever we have a good upper bound on $M^{*}(K), K$ is essentially isotropic. For convenience, we define $M_{2}^{*}(K)=\left(\int_{S^{n-1}}\left(\|\theta\|_{K}^{*}\right)^{2} d \sigma(\theta)\right)^{1 / 2}$, which is well known to be equivalent to $M^{*}(K)$ (by Kahane's inequality for instance).

Proposition 4.5. For any 2-convex body $K$ with constant $\alpha$ and volume 1, we have:

$$
\int_{K}|x| d x \leq C \frac{M^{*}(K)}{\sqrt{\alpha}} .
$$

Proof.

$$
\begin{aligned}
& \int_{K}|x| d x \leq\left(\int_{K}|x|^{2} d x\right)^{1 / 2}=\sqrt{n}\left(\int_{K} \int_{S^{n-1}}\langle x, \theta\rangle^{2} d \sigma(\theta) d x\right)^{1 / 2} \\
= & \sqrt{n}\left(\int_{S^{n-1}} \int_{K}\langle x, \theta\rangle^{2} d x d \sigma(\theta)\right)^{1 / 2}=\sqrt{n}\left(\int_{S^{n-1}}\|\langle\cdot, \theta\rangle\|_{L_{2}(K)}^{2} d \sigma(\theta)\right)^{1 / 2} \\
\leq & C \sqrt{n}\left(\int_{S^{n-1}}\|\langle\cdot, \theta\rangle\|_{L_{\psi_{2}}(K)}^{2} d \sigma(\theta)\right)^{1 / 2} \leq \frac{C^{\prime}}{\sqrt{\alpha}}\left(\int_{S^{n-1}}\left(\|\theta\|_{K}^{*}\right)^{2} d \sigma(\theta)\right)^{1 / 2},
\end{aligned}
$$

where we used Proposition 2.4 in the last inequality. The last term is equal to $\frac{C^{\prime}}{\sqrt{\alpha}} M_{2}^{*}(K)$, which is majorized by $\frac{C^{\prime \prime}}{\sqrt{\alpha}} M^{*}(K)$. 
The last Proposition has an interesting consequence regarding 2Firey sums of 2-convex bodies in minimal mean-width position, or in any bounded $M^{*}$ position in general.

Corollary 4.6. Let $K$ and $T$ be 2-uniformly convex bodies, such that $M_{2}^{*}(K) \leq C_{K}$ Vol.rad. $(K)$ and $M_{2}^{*}(T) \leq C_{T}$ Vol.rad. $(T)$ (and therefore essentially isotropic). Then $M_{2}^{*}\left(K+{ }_{2} T\right) \leq \max \left(C_{K}, C_{T}\right)$ Vol.rad. $\left(K+{ }_{2} T\right)$. In particular, $K+{ }_{2} T$ is essentially isotropic.

Proof. Notice that $\left(M_{2}^{*}\right)^{2}$ is clearly additive with respect to 2-Firey sums, whereas by Lut93] $\left|K+{ }_{2} T\right|^{2 / n} \geq|K|^{2 / n}+|T|^{2 / n}$. The claim then easily follows.

An additional property of any position for which $M^{*}(K)$ is bounded, is that it automatically satisfies half of the conditions of being in a 2regular M-position. Recall that a convex body $K$ in $\mathbb{R}^{n}$ is said to be in $a$-regular M-position $(0<a \leq 2)$ if its homothetic copy $K^{\prime}$, normalized to that $\left|K^{\prime}\right|=\left|D_{n}\right|$, satisfies:

$$
N\left(K^{\prime}, t D_{n}\right) \leq \exp \left(C n / t^{a}\right) \text { and } N\left(\left(K^{\prime}\right)^{\circ}, t D_{n}\right) \leq \exp \left(C n / t^{a}\right),
$$

for $t \geq 1$, where $N(K, L)$ is the covering number of $K$ by $L$ (see GM01) and $C>0$ is a universal constant. It was shown by Pisier ([Pis89]) that an $a$-regular M-position for $0<a<2$ always exists (with a constant $C$ in (4.3) depending only on $a$ ). When $M^{*}(K)$ is bounded and $|K|=\left|D_{n}\right|$, by Sudakov's inequality ([GM01]):

$$
N\left(K, t D_{n}\right) \leq \exp \left(C n\left(M^{*}(K) / t\right)^{2}\right) \leq \exp \left(C n / t^{2}\right)
$$

for $t \geq 1$, so half of the condition for being in a 2-regular M-position is satisfied. In general, the other half of the condition, namely:

$$
N\left(K^{\circ}, t D_{n}\right) \leq \exp \left(C n / t^{2}\right),
$$

does not follow from knowing that $M^{*}(K)$ is bounded. Nevertheless, we mention two cases where this would follow. If $K$ is in minimal meanwidth position and $|K|=\left|D_{n}\right|$, in which case both $M(K)$ and $M^{*}(K)$ are bounded by (4.1), then (4.4) follows from Sudakov's inequality applied to $K^{\circ}$. Another case is when $K$ is in a finite volume-ratio position with bounded $M^{*}(K)$ (remember that we know that $K$ has finite volume-ratio), in which case (4.4) is trivially satisfied. The second case, if it exists, will be preferred over the first, since it adds the finitevolume ratio position property (which is not guaranteed in general by the minimal mean-width position), in particular implying that $M(K)$ is bounded.

Luckily, for a 2-convex body, there exists an "all-in-one" position which gives all of the above mentioned properties: bounded $M^{*}$, having 
finite volume-ratio (and therefore being in a 2-regular M-position) and essential isotropicity. This position is exactly John's maximal-volume ellipsoid position. This follows from the following useful lemma from Mil06a (which appeared first in an equivalent form in [DMTJ81):

Lemma 4.7. For any convex body $K$ in John's maximal-volume ellipsoid position, the following holds:

$$
M_{2}^{*}(K) b(K) \leq T_{2}\left(X_{K}^{*}\right)
$$

where $b(K)=\max _{\theta \in S^{n-1}}\|\theta\|_{K}$.

For a 2-convex body $K$ with constant $\alpha$, the polar body is 2 -smooth with constant $1 /(16 \alpha)$, and therefore by Lemma $4.3, X_{K}^{*}$ has type 2 with constant $T_{2}\left(X_{K}^{*}\right) \leq C / \sqrt{\alpha}$. Noting that $M^{*}(K) \leq M_{2}^{*}(K)$, Lemma 4.7 therefore gives:

Corollary 4.8. A 2-convex body $K$ with constant $\alpha$ in John's maximalvolume ellipsoid position, satisfies:

$$
M^{*}(K) b(K) \leq \frac{C}{\sqrt{\alpha}} .
$$

Since $M^{*}(K) b(K)$ is invariant under homothety, we may assume above that $|K|=\left|D_{n}\right|$, in which case $b(K) \geq 1$ (by volume consideration) and $M^{*}(K) \geq 1$ (by Urysohn's inequality). We therefore see that in John's maximal-volume ellipsoid position $M^{*}(K) \leq$ $C / \sqrt{\alpha}$ Vol.rad. $(K)$. The similar bound on $b$ implies again that $K$ has finite-volume ratio, v.r. $(K) \leq C / \sqrt{\alpha}$, with the same bound (up to a possible constant) as in Proposition 1.3. Proposition 4.5 coupled with the latter bound on $M^{*}(K)$ in John's position, imply Proposition 1.4 stated in the Introduction.

One last additional property that we would like our "all-in-one" position to satisfy is having a small-diameter: if $|K|=\left|D_{n}\right|$, we would like to have $\operatorname{diam}(K) \leq C(n / \log n)^{1 / 2}$. The motivation for this requirement comes from ABP03, where it was shown that if an isotropic 2-convex body has small-diameter in the above sense, then most of its marginals are approximately Gaussian (see ABP03 or Section 5 for more details). It is easy to check that this requirement is indeed satisfied by all the $l_{p}^{n}$ unit balls for $1<p \leq 2$ (normalized to have the appropriate volume).

Unfortunately, the small-diameter requirement is not satisfied for a general 2-convex body in isotropic position, as illustrated by the following: 
Example 4.9. Let:

$$
T=\left\{(x, y) \in \mathbb{R}^{2} ; x^{2}+(|y|+1)^{2} \leq 2\right\} .
$$

The set $T$ is 2-convex with constant c, and has two "cusps", at (1,0) and $(-1,0)$. Denote by $K \subset \mathbb{R}^{n}$ the revolution body of $T$ around the y-axis, namely:

$$
K=\left\{\left(x_{1}, \ldots, x_{n}\right) \in \mathbb{R}^{n} ;\left(\left(x_{1}^{2}+\ldots+x_{n-1}^{2}\right)^{1 / 2}, x_{n}\right) \in T\right\} .
$$

It is easy to check that $K$ is 2-convex with constant $c^{\prime}$. Let $\widetilde{K} \subset \mathbb{R}^{n}$ be an isotropic image of $K$ of volume 1 . Then $\operatorname{diam}(\widetilde{K}) \geq c^{\prime \prime} n$.

Sketch of proof. Around its "cusp" hyperplane $e_{n}^{\perp}, K$ looks like a twosided cone, and therefore half of the volume of $K$ lies inside the slab $\left\{x \in \mathbb{R}^{n} ;\left|\left\langle x, e_{n}\right\rangle\right| \leq c(n) / n\right\}$ with $c(n) \approx 1$. But in isotropic position of volume 1 , half of the volume of $\widetilde{K}$ lies inside slabs of width in the order of $L_{K}$ (and $L_{K} \approx 1$ by Proposition 1.1). This means that we must inflate $K$ by an order of $n$ in the direction of $e_{n}$ when passing to $\widetilde{K}$, implying that $\operatorname{diam}(\widetilde{K}) \geq c^{\prime \prime} n$.

Nevertheless, the following proposition shows that in Löwner's minimal-volume outer ellipsoid position, the small-diameter requirement is satisfied, although we are not able to guarantee any of the other "good" properties satisfied by John's maximal-volume ellipsoid position. We note that $K$ is in Löwner's position iff $K^{\circ}$ is in John's position.

Proposition 4.10. Let $K$ be any 2-convex body with constant $\alpha$ and volume 1 . Then there exists a constant $\lambda>0$ which depends on $\alpha$ only, such that in Löwner's minimal-volume outer ellipsoid position, $\operatorname{diam}(K) \leq \frac{C}{\lambda} n^{1 / 2-\lambda}$.

Proof. Apply Lemma 4.7 to $K^{\circ}$, which by duality is in John's maximalvolume ellipsoid position. Then:

$$
M_{2}(K) \operatorname{diam}(K) \leq T_{2}\left(X_{K}\right) .
$$

Since $M_{2}(K) \geq$ Vol.rad. $(K)^{-1}=1$ by Jensen's inequality, it is enough to show that $T_{2}\left(X_{K}\right)$ is bounded by $C n^{1 / 2-\lambda}$. By Lemma 4.4, we know that there exists a $p>1$ which depends on $\alpha$ only, such that $T_{p}\left(X_{K}\right) \leq$ $1 /(p-1)$, so it remains to pass from type- $p$ to type- 2 . But this is an easy consequence of a result by Tomczak-Jaegermann ([TJ79]), who showed that it is enough to evaluate the type 2 constant of an $n$-dimensional Banach space on $n$ vectors. If $x_{1} \ldots x_{n}$ is any sequence in $\mathbb{R}^{n}$, then by 
Hölder's inequality:

$$
\mathbb{E}\left\|\sum_{i=1}^{n} \varepsilon_{i} x_{i}\right\|_{K} \leq \frac{1}{p-1}\left(\sum_{i=1}^{n}\left\|x_{i}\right\|_{K}^{p}\right)^{\frac{1}{p}} \leq \frac{n^{\frac{1}{p}-\frac{1}{2}}}{p-1}\left(\sum_{i=1}^{n}\left\|x_{i}\right\|_{K}^{2}\right)^{\frac{1}{2}} .
$$

Therefore $T_{2}\left(X_{K}\right) \leq \frac{C}{\lambda} n^{1 / 2-\lambda}$, for $\lambda=1-1 / p$.

We conclude this section by mentioning that the results of Section 2 imply that for 2-convex bodies, the isotropic position is a 1-regular M-position. Indeed, since the isotropic position is also a finite volumeratio position, the second half of condition (4.3) is trivially satisfied. The first half is satisfied by the result from ( $\mathrm{Har03}$. or Kla05, Proposition 5.4]), which shows that this is always the case for any isotropic body for which $L_{K}$ is bounded. Note that [GM98, Theorem 5.6] (which uses Dudley's entropy bound) enables us to bound the mean-width of a convex body in an $a$-regular M-position, which for a 1-regular position gives:

$$
M^{*}(K) \leq C \operatorname{diam}(K)^{1 / 2} \operatorname{Vol.rad} .(K)^{1 / 2} .
$$

Since $\operatorname{diam}(K) \leq C \sqrt{n} L_{K}$ Vol.rad. $(K)$ in isotropic position (e.g. [MP88]), we conclude that $M^{*}(K) \leq C(\alpha) n^{1 / 4} \operatorname{Vol} . r a d$. $(K)$ for any 2-convex body $K$ with constant $\alpha$ in isotropic position. It is still unclear to us whether the isotropic position is always a 2-regular M-position, which would imply (as above) that $M^{*}(K) \leq C(\alpha) \log (n)$ Vol.rad. $(K)$.

To summarize, we have seen the following implications for a 2-convex body:

- Minimal mean-width position implies essential isotropicity and a 2-regular M-position.

- John's maximal-volume ellipsoid position implies finite volumeratio position, essential minimal mean-width, 2-regular M-position and essential isotropicity.

- Löwner's minimal-volume outer ellipsoid position implies "smalldiameter".

- Isotropic position implies finite volume-ratio position and 1regular M-position.

\section{Gaussian marginals}

Similarly to the 2 -convex case, we say that a convex body $K$ is $p$ convex (with constant $\alpha$ ) if its modulus of convexity satisfies $\delta_{K}(\epsilon) \geq$ $\alpha \epsilon^{p}$ for all $\epsilon \in(0,2)$. Let us also denote $d_{K}=\operatorname{diam}(K)$. It is wellknown and easy to see (e.g. Led01] or follow the argument in Lemma 
2.2) that the Gromov-Milman Theorem (2.2) immediately implies the following:

Lemma 5.1. Let $K$ be a p-convex body with constant $\alpha$ and of volume 1. For any 1-Lipschitz function $f$ on $K$ denote by Med $(f)$ the median of $f$, i.e. the value for which $\operatorname{Vol}\{x \in K ; f(x) \geq \operatorname{Med}(f)\} \geq 1 / 2$ and $\operatorname{Vol}\{x \in K ; f(x) \leq \operatorname{Med}(f)\} \geq 1 / 2$. Then:

$$
\operatorname{Vol}\{x \in K ; f(x) \geq \operatorname{Med}(f)+t\} \leq 2 \exp \left(-2 \alpha n\left(t / d_{K}\right)^{p}\right) .
$$

Let us denote $E(f)=\int_{K} f(x) d x$. As in ABP03, we deduce from Lemma 5.1 that $|E(f)-M e d(f)| \leq C d_{K}(\alpha n)^{-\frac{1}{p}}$. We therefore have: $\mathrm{Vol}\left\{x \in K ;|f(x)-E(f)| \geq t+C d_{K}(\alpha n)^{-\frac{1}{p}}\right\} \leq 4 \exp \left(-2 \alpha n\left(\frac{t}{d_{K}}\right)^{p}\right)$, and it is easy to check that this implies:

Lemma 5.2. With the same notations as in Lemma 5.1:

$$
\operatorname{Vol}\{x \in K ;|f(x)-E(f)| \geq t\} \leq 4 \exp \left(-2 c^{p} \alpha n\left(\frac{t}{d_{K}}\right)^{p}\right) .
$$

Using this, it was shown in ABP03 that if $K$ is an isotropic $p$-convex body (with constant $\alpha$ ) with $|K|=1$ and $\operatorname{diam}(K) \leq R \sqrt{n}$, then:

$$
\operatorname{Vol}\left\{x \in K ;\left|\frac{|x|}{\sqrt{n}}-L_{K}\right| \geq R t\right\} \leq 4 \exp \left(-2 c^{p} \alpha n t^{p}\right) \text {. }
$$

Choosing $t=C\left(\frac{\log (n)}{\alpha n}\right)^{1 / p}$, this implies:

$$
\operatorname{Vol}\left\{x \in K ;\left|\frac{|x|}{\sqrt{n}}-L_{K}\right| \geq C R\left(\frac{\log (n)}{\alpha n}\right)^{1 / p}\right\} \leq \frac{1}{n} .
$$

The authors of ABP03 conclude that if $R \ll(\alpha n / \log (n))^{1 / p}$, (5.1) implies a concentration of the volume of $K$ inside a spherical shell around a radius of $\sqrt{n} L_{K}$. It was shown in ABP03 that such a concentration implies that most marginals of the uniform distribution on $K$ will have an approximately Gaussian distribution (see Theorem 5.3 below). Unfortunately, our investigation of the case $p=2$ shows that this condition on $R$ is not satisfied in general by isotropic 2-convex bodies, as demonstrated by Example 4.9. Nevertheless, Proposition 4.10 shows that in Löwner's minimal-volume ellipsoid position, we do have $R \leq C n^{1 / 2-\lambda} / \lambda$ where $\lambda$ depends only on the 2-convexity constant of $K$. In this case, the concentration result of ABP03] still holds, with the minor change that $L_{K}$ in (5.1) is replaced by $\int_{K}|x| d x / \sqrt{n}$ (note that this value is always greater than $c_{1} L_{K} \geq c_{2}$, e.g. [MP88]). Although $K$ is no longer isotropic, it is possible to generalize the argument in 
ABP03 to a body in arbitrary position. This is done in Mil06b, where the following is shown:

Theorem 5.3 (Generalization of ABP03). Let $K$ be a centrallysymmetric convex body in $\mathbb{R}^{n}$ of volume 1 , and assume that for some $\rho>0$ and $\epsilon<1 / 2$ :

$$
\operatorname{Vol}\left\{x \in K ;\left|\frac{|x|}{\sqrt{n}}-\rho\right| \geq \epsilon \rho\right\} \leq \epsilon .
$$

For $\theta \in S^{n-1}$ denote $g_{\theta}(s)=\operatorname{Vol}\left(K \cap\left\{s \theta+\theta^{\perp}\right\}\right)$ and let $\rho_{\theta}^{2}=\int_{-\infty}^{\infty} s^{2} g_{\theta}(s) d s$.

Denote the Gaussian density with variance $\rho^{2}$ by $\phi(s)=\frac{1}{\sqrt{2 \pi} \rho} \exp \left(-\frac{s^{2}}{2 \rho^{2}}\right)$ and let $H(\theta)=\sup _{t>0}\left|\int_{-t}^{t} g_{\theta}(s) d s-\int_{-t}^{t} \phi(s) d s\right|$. Then for any $0<\delta<$ $c$ :

$$
\begin{aligned}
& \sigma\left\{\theta \in S^{n-1} ; H(\theta) \leq \delta+4 \epsilon+\frac{c_{1}}{\sqrt{n}}\right\} \\
& \quad \geq 1-C_{1} C_{i s o}(K) \sqrt{n} \log n \exp \left(-\frac{c_{2} n \delta^{2}}{C_{i s o}(K)^{2}}\right),
\end{aligned}
$$

where:

$$
\rho_{\text {max }}=\max _{\theta \in S^{n-1}} \rho_{\theta}, \rho_{\text {avg }}=\int_{S^{n-1}} \rho_{\theta} d \sigma(\theta), C_{i s o}(K)=\frac{\rho_{\max }}{\rho_{\text {avg }}} .
$$

Remark 5.4. As usual, it is easy to verify that $\rho_{\text {avg }}$ and $\rho$ above are equivalent to within absolute constants (since $\epsilon<1 / 2$ ).

If $T$ is a volume preserving linear transformation such that $\widetilde{K}=$ $T(K)$ is isotropic, then clearly $\rho_{\max }=\left\|T^{-1}\right\|_{o p} L_{K}$, where $\|\cdot\|_{o p}$ denotes the operator norm. Since $\rho_{\text {avg }}^{2} \approx \frac{1}{n} \int_{K}|x|^{2} d x \geq L_{K}^{2}$ (e.g. [MP88]), it follows that $C_{i s o}(K) \leq C\left\|T^{-1}\right\|_{o p}$. Hence, knowing that $r D_{n} \subset \widetilde{K}$ and $K \subset R D_{n}$ would imply that $C_{i s o}(K) \leq C R / r$. By Lemma 2.3 and Proposition 4.10. $c \sqrt{\alpha} \sqrt{n} L_{K} D_{n} \subset \widetilde{K}$ and $K \subset C n^{1-\lambda} / \lambda$ in Löwner's position, where $\lambda>0$ depends only on $\alpha$. We therefore have in this position:

$$
C_{i s o}(K) \leq \min \left(\frac{C n^{1 / 2-\lambda}}{\sqrt{\alpha} \lambda L_{K}}, C \sqrt{n}\right) .
$$

Hence, regardless of its a-priori diameter, by putting a 2-convex body $K$ with constant $\alpha$ in Löwner's position, we deduce by Proposition 4.10. Lemma 5.2 and Theorem $[5.3$ that most marginals of $K$ are approximately Gaussian in the above sense, where the level of proximity ( $\epsilon$ above) depends only on $\alpha$. Summarizing, we have: 
Theorem 5.5. Let $K$ be a 2-convex body with constant $\alpha$ and volume 1. Assume that $K$ is in Löwner's minimal-volume outer ellipsoid position. Then with the same notations as in Theorem 5.3 and with $\rho=\int_{K}|x| d x / \sqrt{n}$, we have for any $0<\delta<c$ :

$$
\sigma\left\{\theta \in S^{n-1} ; H(\theta) \leq \delta+4 \epsilon+\frac{c_{1}}{\sqrt{n}}\right\} \geq 1-n^{5 / 2} \exp \left(-c_{2} \alpha n^{2 \lambda} \lambda^{2} \delta^{2}\right),
$$

where $\epsilon=C \sqrt{\log n} \alpha^{-1 / 2} \lambda^{-1} n^{-\lambda}$ and $\lambda=\lambda(\alpha)>0$ depends on $\alpha$ only.

Before concluding, we remark that placing a 2-convex body $K$ in Löwner's position is just a convenient "pre-processing" step. In fact, in any position we always have at least one approximately Gaussian marginal (in the above sense); it just happens that in Löwner's position we can show this for "most" marginals w.r.t. the Haar probability measure on the unit sphere, and this would equally be true in an arbitrary position by choosing a different measure (the one induced by the change of positions, for example). The reason is that the metric given by $H(\theta)$ in Theorem 5.3 is invariant under volume-preserving linear transformations. More precisely, given such a $T$, and any body $K$ and $\rho>0$, it is immediate to check that:

$$
\int_{-t}^{t}\left(g_{\theta}^{K}(s)-\phi_{\rho}(s)\right) d s=\int_{-\frac{t}{|T(\theta)|}}^{\frac{t}{T(\theta) \mid}}\left(g_{\frac{T(\theta)}{T(\theta) \mid}}^{T(K)}(s)-\phi_{\rho|T(\theta)|}(s)\right) d s,
$$

so by Theorem [5.5 we can control the supremum over $t>0$ of either expressions for at least one $\theta \in S^{n-1}$ if $K$ is a 2-convex body in Löwner's position and $\rho=\int_{K}|x| d x / \sqrt{n}$.

\section{REFERENCES}

[ABP03] M. Anttila, K. Ball, and I. Perissinaki, The central limit problem for convex bodies, Trans. Amer. Math. Soc. 355 (2003), no. 12, 4723-4735.

[AdRBV98] J. Arias-de Reyna, K. Ball, and R. Villa, Concentration of the distance in finite-dimensional normed spaces, Mathematika 45 (1998), no. 2, $245-252$.

[BKM03] J. Bourgain, B. Klartag, and V. Milman, Symmetrization and isotropic constants of convex bodies, Geometric Aspects of Functional Analysis, Lecture Notes in Mathematics, vol. 1850, Springer, 2002-2003, pp. 101115.

[BL00a] Y. Benyamini and J. Lindenstrauss, Geometric nonlinear functional analysis. Vol. 1, American Mathematical Society Colloquium Publications, vol. 48, American Mathematical Society, Providence, RI, 2000.

[BL00b] S. G. Bobkov and M. Ledoux, From Brunn-Minkowski to BrascampLieb and to logarithmic Sobolev inequalities, Geom. Funct. Anal. 10 (2000), no. 5, 1028-1052. 
[BM87] J. Bourgain and V. D. Milman, New volume ratio properties for convex symmetric bodies in $\mathbb{R}^{n}$, Inventiones Mathematicae 88 (1987), 319340 .

[BTV00] O. Blasco, V. Tarieladze, and R. Vidal, K-convexity and duality for almost summing operators, Georgian Math. J. 7 (2000), no. 2, 245-268.

[DMTJ81] W. J. Davis, V. D. Milman, and N. Tomczak-Jaegermann, The distance between certain $n$-dimensional banach spaces, Israel Journal of Mathematics 39 (1981), 1-15.

[FTJ79] T. Figiel and N. Tomczak-Jaegermann, Projections onto Hilbertian subspaces of Banach spaces, Israel J. Math. 33 (1979), no. 2, 155-171.

[GM87] M. Gromov and V. D. Milman, Generalization of the spherical isoperimetric inequality to uniformly convex Banach spaces, Compositio Math. 62 (1987), no. 3, 263-282.

[GM98] A. A. Giannopoulos and V. D. Milman, Mean width and diameter of proportional sections of a symmetric convex body, J. Reine Angew. Math. 497 (1998), 113-139.

[GM01] A. A. Giannopoulos and V. D. Milman, Euclidean structure in finite dimensional normed spaces, Handbook of the geometry of Banach spaces, Vol. I (Amsterdam), North-Holland, Amsterdam, 2001, pp. 707-779.

[GP04] O. Guédon and G. Paouris, Concentration of mass on the schatten classes, Manuscript, 2004.

[Har03] M. Hartzoulaki, Probabilistic methods in the theory of convex bodies, Ph.D. thesis, University of Crete, March 2003.

[Hen80] D. Hensley, Slicing convex bodies: Bounds of slice area in terms of the body's covariance, Proc. Amer. Math. Soc. 79 (1980), 619-625.

[JSZ85] W. B. Johnson, G. Schechtman, and J. Zinn, Best constants in moment inequalities for linear combinations of independent and exchangeable random variables, Ann. Probab. 13 (1985), no. 1, 234-253.

[Jun94] M. Junge, Hyperplane conjecture for quotient spaces of $l_{p}$, Forum Math. 6 (1994), 617-635.

[Kaš77] B. S. Kašin, The widths of certain finite-dimensional sets and classes of smooth functions, Izv. Akad. Nauk SSSR Ser. Mat. 41 (1977), no. 2, 334-351, 478.

[Kla05] B. Klartag, An isomorphic version of the slicing problem, J. Funct. Anal. 218 (2005), no. 2, 372-394.

[KMP98] H. König, M. Meyer, and A. Pajor, The isotropy constants of the Schatten classes are bounded, Math. Ann. 312 (1998), no. 4, 773-783.

[Led01] M. Ledoux, The concentration of measure phenomenon, Mathematical Surveys and Monographs, vol. 89, American Mathematical Society, Providence, RI, 2001.

[LT79] J. Lindenstrauss and L. Tzafriri, Classical Banach spaces. II, Ergebnisse der Mathematik und ihrer Grenzgebiete [Results in Mathematics and Related Areas], vol. 97, Springer-Verlag, Berlin, 1979, Function spaces.

[Lut93] E. Lutwak, The Brunn-Minkowski-Firey theory. I. Mixed volumes and the Minkowski problem, J. Differential Geom. 38 (1993), no. 1, 131150 . 
[Mil06a] E. Milman, Dual mixed volumes and the slicing problem, to appear in Advances in Mathematics, www.arxiv.org/math.FA/0512207, 2006.

[Mil06b] E. Milman, On gaussian marginals of uniformly convex bodies, Manuscript, 2006.

[MP76] B. Maurey and G. Pisier, Séries de variables aléatoires vectorielles indépendantes et propriétés géométriques des espaces de Banach, Studia Math. 58 (1976), no. 1, 45-90.

[MP88] V. D. Milman and A. Pajor, Isotropic position and interia ellipsoids and zonoids of the unit ball of a normed n-dimensional space, Geometric Aspects of Functional Analysis, Lecture Notes in Mathematics, vol. 1376, Springer-Verlag, 1987-1988, pp. 64-104.

[MS86] V. D. Milman and G. Schechtman, Asymptotic theory of finitedimensional normed spaces, Lecture Notes in Mathematics, vol. 1200, Springer, Berlin, 1986.

[Nor60] G. Nordlander, The modulus of convexity in normed linear spaces, Ark. Mat. 4 (1960), 15-17.

[Pao02] G. Paouris, $\psi_{2}$-estimates for linear functionals on zonoids, Geometric Aspects of Functional Analysis, Lecture Notes in Mathematics, vol. 1807, Springer, 2001-2002, pp. 211-222.

[Pis73] G. Pisier, Sur les espaces de Banach qui ne contiennent pas uniformément de $l_{n}^{1}$, C. R. Acad. Sci. Paris Sér. A-B 277 (1973), A991A994.

[Pis89] G. Pisier, A new approach to several results of V. Milman, J. Reine Angew. Math. 393 (1989), 115-131.

[San49] L. A. Santaló, An affine invariant for convex bodies of $n$-dimensional space, Portugaliae Math. 8 (1949), 155-161.

[Sch95] M. Schmuckenschläger, A concentration of measure phenomenon on uniformly convex bodies, Geometric aspects of functional analysis (Israel, 1992-1994) (Basel), Oper. Theory Adv. Appl., vol. 77, Birkhäuser, Basel, 1995, pp. 275-287.

[STJ80] S. Szarek and N. Tomczak-Jaegermann, On nearly Euclidean decomposition for some classes of Banach spaces, Compositio Math. 40 (1980), no. 3, 367-385.

[Sza80] S. Szarek, Volume estimates and nearly Euclidean decompositions for normed spaces, Seminar on Functional Analysis, 1979-1980 (French) (Palaiseau), École Polytech., Palaiseau, 1980, pp. Exp. No. 25, 8.

[TJ74] N. Tomczak-Jaegermann, The moduli of smoothness and convexity and the Rademacher averages of trace classes $S_{p}(1 \leq p<\infty)$, Studia Math. 50 (1974), 163-182.

[TJ79] N. Tomczak-Jaegermann, Computing 2-summing norm with few vectors, Ark. Mat. 17 (1979), no. 2, 273-277. 